\title{
Holographic bottomonium formation in a cooling strong-interaction medium at finite baryon density
}

\author{
R. Zöllner@ and B. Kämpfer \\ Helmholtz-Zentrum Dresden-Rossendorf, 01314 Dresden, Germany and Institut für Theoretische Physik, \\ TU Dresden, 01062 Dresden, Germany
}

(Received 20 September 2021; accepted 13 October 2021; published 11 November 2021)

\begin{abstract}
The shrinking of the bottomonium spectral function towards narrow quasiparticle states in a cooling strong-interaction medium at finite baryon density is followed within a holographic bottom-up model. The five-dimensional Einstein-dilaton-Maxwell background is adjusted to the lattice-QCD results of sound velocity and susceptibilities. The zero-temperature bottomonium spectral function is adjusted to the experimental $\Upsilon$ ground state mass and the first radial excitations. At baryo-chemical potential $\mu_{B}=0$, these two pillars let emerge the narrow quasiparticle state of the $r$ ground state at a temperature of about $150 \mathrm{MeV}$. Excited states are consecutively formed at lower temperatures by about 10 (20) MeV for the $2 S(3 S)$ vector states. The baryon density, i.e., $\mu_{B}>0$, pulls that formation pattern to lower temperatures. At $\mu_{B}=200 \mathrm{MeV}$, we find a shift by about $15 \mathrm{MeV}$.
\end{abstract}

DOI: 10.1103/PhysRevD.104.106005

\section{INTRODUCTION}

The observation of sequential bottomonium suppression [1-5] in relativistic heavy-ion collisions at LHC has sparked a series of dedicated investigations, e.g., [6-14]. Such heavy-quark flavor degrees of freedom receive currently some interest as valuable probes of hot and dense strong-interaction matter produced in heavy-ion collisions at LHC energies. The information encoded, e.g., in heavy quarkonia $(\bar{Q} Q=c \bar{c}$ or $b \bar{b})$ observables, supplements penetrating electromagnetic probes and hard (jet) probes and the rich flow observables, thus complementing each other in characterizing the dynamics of quarks and gluons up to the final hadronic states (cf. contributions in [15] for the state of the art). Heavy quarks emerge essentially in early, hard processes, that is, they witness the course of a heavy-ion collision-either as individual entities or as subjects of dissociating and regenerating bound states. Accordingly, the heavy-quark physics address such issues as charm $(c, \bar{c})$ and bottom $(b, \bar{b})$ dynamics related to transport coefficients [14,16-21] in the rapidly evolving and highly anisotropic ambient quark-gluon medium $[22,23]$ as well as $\bar{Q} Q$ states as open quantum systems [24-27]. The wealth of experimental data from LHC, and also from RHIC, enables a tremendous refinement of our understanding of heavy-quark dynamics. For a recent

Published by the American Physical Society under the terms of the Creative Commons Attribution 4.0 International license. Further distribution of this work must maintain attribution to the author(s) and the published article's title, journal citation, and DOI. Funded by SCOAP ${ }^{3}$. survey on quarkonium physics we refer the interested reader to [28].

The yields of various hadron species, light nuclei and antinuclei emerging from heavy-ion collisions at LHC energies are well described by the thermostatistical hadronization model $[29,30]$ over an interval of nine orders of magnitude. The final hadrons and nuclear clusters are determined by two parameters: the freeze-out temperature $T_{f o} \approx 155 \mathrm{MeV}$ and a freeze-out volume depending on the system size or centrality of the collision. Due to the nearperfect matter-antimatter symmetry at top LHC energies, the baryo-chemical potential $\mu_{B}$ is exceedingly small, $\mu_{B} / T_{f o} \ll 1$. While the authors of [31] see a delicate interplay of elastic and inelastic hadron reactions as a governing principle of the hadrochemical freeze-out, it is argued in [30] that the freeze-out of color-neutral objects happens just in the demarcation region of hadron matter to quark-gluon plasma, i.e., confined vs deconfined stronginteraction matter. In fact, lattice-QCD results report a pseudocritical temperature of $T_{\mathrm{pc}}=(156 \pm 1.5) \mathrm{MeV}$ [32] and (158.0 \pm 0.6$) \mathrm{MeV}$ [33] — values agreeing with the disappearance of the chiral condensates and the maximum of some susceptibilities. The key is the adjustment of physical quark masses and the use of $2+1$ flavors [34,35], in short $\mathrm{QCD}_{2+1}$ (phys). Details of the coincidence of deconfinement and chiral symmetry restoration are matters of debate [36]. Reference [37] advocates flavor-dependent freeze-out temperatures. Note that at $T_{\mathrm{pc}}$ no phase transition happens, rather the thermodynamics are characterized by a crossover accompanied by a pronounced nearby minimum of the sound velocity. This situation continues to nonzero baryon density as long as the baryo-chemical potential $\mu_{B}$ is small, $\mu_{B} / T_{\mathrm{pc}} \ll 1$. 
Among the tools for describing hadrons as composite strong-interaction systems is holography. Anchored in the famous AdS/CFT correspondence, holographic bottom-up approaches have facilitated a successful description of mass spectra, coupling strengths/decay constants etc. of various hadron species. While the direct link to QCD by a holographic QCD-dual or rigorous top-down formulations are still missing, one has to restrict the accessible observables to explore certain frameworks and scenarios. We consider here a framework which merges for the first time (i) $\mathrm{QCD}_{2+1}$ (phys) thermodynamics described by a dynamical holographic gravity-dilaton-Maxwell background and (ii) holographic probe quarkonia. We envisage a scenario which embodies QCD thermodynamics of $\mathrm{QCD}_{2+1}$ (phys) and the emergence of hadron states at $T_{c}$ at the same time. One motivation of our work is the exploration of a holographic model which is in agreement with the above hadron phenomenology in heavy-ion collisions at LHC energies. Early holographic studies [38-40] to hadrons at finite temperatures faced the problem of meson melting at temperatures significantly below the deconfinement temperature $T_{\mathrm{pc}}$. Several proposals have been made [41-43] to find rescue avenues which accommodate hadrons at and below $T_{\mathrm{pc}}$. Otherwise, a series of holographic models of hadron melting without reference to realistic QCD thermodynamics, e.g., [44-52] — mostly with emphasis on quarkonium melting - finds quarkonia states well above, at and below $T_{\mathrm{pc}}$ in agreement with lattice-QCD results [53-56]. It is therefore tempting to account for the proper QCD-related background.

In the temperature region $T=\mathcal{O}\left(T_{\mathrm{pc}}\right)$, the impact of charm and bottom degrees of freedom on the quark-gluon hadron thermodynamics is minor [57]. Thus, we consider quarkonia, in particular bottomonium, as test particles. We follow [58-61] and model the holographic background by a gravity-dilaton setup supplemented by a Maxwell field $[62,63]$, i.e., without adding further fundamental degrees of freedom to the dilaton. That is, the dilaton potential and its coupling to the Maxwell field are adjusted to $\mathrm{QCD}_{2+1}$ (phys) lattice data. Our emphasis is here on the formation of bottomonium in a cooling strong-interaction environment. Thereby, the bottomonium properties are described by a spectral function. The primary aim of the present paper is to study the impact of a finite baryon density of the strong-interaction medium, thus complementing $[64,65]$. Finite baryon effects become relevant at smaller beam energies, e.g., at RHIC, and are systematically accessible in the beam energy scans [66-68]. We restrict ourselves to equilibrium and leave nonequilibrium effects, e.g., [69,70], for future work.

Such $\mu_{B}>0$ effects on holographic bottomonium spectroscopy have been considered, e.g., in [44,51,71]. Our present investigation is distinguished by choosing a holographic bottom-up background which is adjusted to QCDlattice data of sound velocity and susceptibilities in the temperature range $100 \mathrm{MeV}<T<600 \mathrm{MeV}$. That is, the gravity-dilaton-Maxwell fields are dynamically determined by solutions of the Einstein equations consistent with the equations of motion of dilaton and Maxwell fields. We do not touch the large- $\mu_{B}$ region or a conjectured critical point $[62,63,72-76]$ since the experimental access to bottomonium physics is expected to be feasible at not too small beam energies, i.e., at low values of $\mu_{B}$ in the central rapidity region.

Our paper is organized as follows. In Sec. II, the dynamics of the probe quarkonia is formulated, and the coupling to the thermodynamics-related background is explained in Sec. III. Both ingredients are joined in Sec. IV for the calculation of the spectral functions. The numerical results for the bottomonium states $\Upsilon(1 S, 2 S, 3 S)$ are presented in Sec. V. We summarize in Sec. VI. Appendix A details the field equations for the Einsteindilaton-Maxwell model with the radial bulk coordinate $z$. Appendix B considers some options for UV-IR matching to generate the $\Upsilon(n S) I^{G}\left(J^{P C}\right)=0^{-}\left(1^{--}\right)$spectrum within holography.

\section{BOTTOM-UP MODEL FOR QUARKONIA}

In thermal equilibrium, the admixture of equilibrated heavy quarks in strong-interaction matter at $T<250 \mathrm{MeV}$ is small [57,77]. Rather, initial hard parton interactions (essentially gluon fusion) create heavy quarks in heavy-ion collisions. Thus, heavy-quark pairs serve as test particles and do not need to be back-reacted. In particular, quarkonia constituents are decoupled from the ambient quark content, with the exception of the gluon component. In a model with minimalistic field content one would prefer to keep the effective gravity-dilaton background (extended by the Maxwell field $\mathcal{B}$ for mimicking $\mu_{B}>0$ ) to catch QCD thermodynamics and attribute to the test particles solely one vector field $\mathcal{A}$. A $U(1)$ gauge field $\mathcal{A}(z)$ in the bulk is supposed to be the dual of the vector meson current operator $\bar{Q} \gamma^{\mu} Q$ at the boundary. The string-frame action is accordingly

$S_{m}^{V}=\frac{1}{k_{V}} \int \mathrm{d}^{4} x \mathrm{~d} z \sqrt{g_{5}} \frac{1}{4} e^{-\phi_{m}} F_{\mathcal{A}}^{2}, \quad \phi_{m}:=\phi-\log G_{m}(\phi)$,

where $F_{\mathcal{A}}$ stands for the Abelian field-strength tensor of $\mathcal{A}$ and $k_{V}=\frac{N_{c}}{24 \pi^{2}}$ with number $N_{c}=3$ of colors. The metric determinant $g_{5}$ is related to the ansatz of the infinitesimal line element

$$
\begin{aligned}
\mathrm{d} s^{2} & =g_{M N} \mathrm{~d} x^{M} \mathrm{~d} x^{N} \\
& :=\exp \left\{A\left(z, z_{H}\right)\right\}\left[f\left(z, z_{H}\right) \mathrm{d} t^{2}-\mathrm{d} \vec{x}^{2}-\frac{\mathrm{d} z^{2}}{f\left(z, z_{H}\right)}\right],
\end{aligned}
$$


with warp function $A$ with AdS asymptotics (see Appendix A for details) and blackening function $f$ which steers the temperature of the system by encoding a dual black hole.

In contrast to common previous practices, the background quantities $g_{5}$ as well as $\phi$ (dilaton field) and $\mathcal{B}$ (Maxwell field) are universal for any test particle, therefore, $G_{m}$ encodes solely the essential properties of the respective test particle. We attribute the quarkonia masses to the considered test particle. Rather than including the heavy-quark masses explicitly, we encode them in the following manner in $G_{m}$. From the the ansatz $\mathcal{A}_{\mu}=$ $\epsilon_{\mu} \varphi(z) \exp \left\{i p_{\nu} x^{\nu}\right\}$ with $\mu, \nu=0, \ldots, 3$, which uniformly separates the $z$ dependence of the gauge field by the bulk-to-boundary propagator $\varphi$ for all components of $\mathcal{A}$, and the constant polarization vector $\epsilon_{\mu}$ and gauges $\mathcal{A}_{z}=0$ and $\partial^{\mu} \mathcal{A}_{\mu}=0$, the equation of motion follows from the action (1) as

$$
\begin{aligned}
\varphi^{\prime \prime} & +\left[\frac{1}{2} A^{\prime}+\left(\partial_{\phi} \log G_{m}-1\right) \phi^{\prime}+(\log f)^{\prime}\right] \varphi^{\prime} \\
& +\frac{p^{\mu} p_{\mu}}{f^{2}} \varphi=0
\end{aligned}
$$

which is cast in the form of a one-dimensional Schrödinger equation with the tortoise coordinate $\xi$,

$\left[\partial_{\xi}^{2}-\left(U(z(\xi))-m_{n}^{2}\right)\right] \psi_{n}(\xi)=0, \quad n=0,1,2, \ldots$,

by the transformation $\psi(\xi)=\varphi(z(\xi)) \exp \left\{\frac{1}{2} \int_{0}^{\xi} \mathrm{d} z \mathcal{S}(\xi)\right\}$ and $p^{\mu} p_{\mu} \rightarrow m_{n}^{2}$. One has to employ $z(\xi)$ from solving $\partial_{\xi}=(1 / f) \partial_{z}$. The Schrödinger-equivalent potential in (4) is

$$
U:=\left(\frac{1}{2} \mathcal{S}^{\prime}+\frac{1}{4} \mathcal{S}^{2}\right) f^{2}+\frac{1}{2} \mathcal{S} f f^{\prime}
$$

as a function of $\xi(z)$ with

$$
\mathcal{S}:=\frac{1}{2} A^{\prime}-\phi^{\prime}+\partial_{z} \log G_{m}(\phi(z)) .
$$

A prime means the derivative with respect to the bulk coordinate $z$.

At $T=0$, we have $f=1$ and $\xi=z$, and $m_{n}$ in Eq. (4) is the quarkonium mass spectrum to be used as input. Therefore, the Schrödinger-equivalent potential $U(z)$ must be chosen in such a manner to deliver the wanted values of $m_{n}$. With given $U(z)$, the Ricatti equation (5) must be solved for $\mathcal{S}$, which in turn determines the heavy-quark mass-specific function $G_{m}(\phi)$ via Eq. (6). This $G_{m}(\phi)$ is assumed as independent of temperature and baryochemical potential, i.e., is ready for direct use at $T>0$ and $\mu_{B}>0$ as well.

In the described chain of operations for getting $G_{m}$, the zero-temperature background quantities $A(z)$ and $\phi(z)$ are needed. They are determined by the temperature independent dilation potential $V(\phi)$, which is adjusted to latticeQCD thermodynamics data, briefly recalled in the next section.

\section{BACKGROUND GENERATED BY THE EINSTEIN-DILATON-MAXWELL BOTTOM-UP MODEL}

We closely follow here the Einstein-dilaton-Maxwell (EdM) model of [75], see also [73,74,76]. The EdM action reads

$$
\begin{aligned}
S_{\mathrm{Edm}}= & \frac{1}{2 \kappa_{5}^{2}} \int \mathrm{d}^{4} x \mathrm{~d} z \sqrt{g_{5}}\left(R-\frac{1}{2} \partial^{M} \phi \partial_{M} \phi-V(\phi)\right. \\
& \left.-\frac{1}{4} \mathcal{F}(\phi) F_{\mathcal{B}}^{2}\right)+S_{\mathrm{GH}},
\end{aligned}
$$

where $R$ is the Einstein-Hilbert part, $F_{\mathcal{B}}^{M N}=\partial^{M} \mathcal{B}^{N}-$ $\partial^{N} \mathcal{B}^{M}$ stands for the field-strength tensor of Abelian gauge field $\mathcal{B}$ à la Maxwell with $\mathcal{B}_{M} \mathrm{~d} x^{M}=\Phi(z) d t$ defining the electrostatic potential, and $\phi$ is a real scalar (dilatonic) field with self-interaction described by the potential $V(\phi)$. The bulk Maxwell field is sourced by the conserved light-quark baryon current $\bar{q} \gamma^{\mu} q$ at the boundary. In such a manner, this field is related to baryon density effects, parametrized by $\mu_{B}$. The Maxwell field and dilaton are coupled by the dynamical strength function $\mathcal{F}(\phi)[62,63]$ (note the analog structures $\frac{1}{4} e^{-\phi_{m}} F_{\mathcal{A}}^{2}$ and $\frac{1}{4} \mathcal{F}(\phi) F_{\mathcal{B}}^{2}$ in the actions (1) and (7) describing the coupling of the dilaton background to the respective vector fields). The Gibbons-Hawking term $S_{\mathrm{GH}}$ for a consistent formulation of the variational problem is not needed explicitly in our context. The numerical value of the "Einstein constant" $\kappa_{5}^{2}=8 \pi G_{N}$ is irrelevant in our context. We relegate the field equations following from the action (7) in the coordinates (2) to Appendix A, but mention here the employed parametrizations

$$
L^{2} V(\phi)=\left\{\begin{array}{l}
-12 \exp \left\{\frac{1}{2} a_{1} \phi^{2}+\frac{1}{4} a_{2} \phi^{4}\right\} \text { for } \phi<\phi_{x}, \\
a_{10} \cosh \left[a_{4}\left(\phi-a_{5}\right)\right]^{a_{3} / a_{4}} \exp \left\{a_{6} \phi+\frac{a_{7}}{a_{8}} \tanh \left[a_{8}\left(\phi-a_{9}\right)\right]\right\} \text { for } \phi>\phi_{x},
\end{array}\right.
$$




$$
\mathcal{F}(\phi)=c_{0}+c_{1} \tanh \left[c_{2}\left(\phi-c_{3}\right)\right]+c_{4} \exp \left\{-c_{5} \phi\right\},
$$

and refer the interested reader to [75] for listings of the parameters $a_{1, \ldots, 10}, \phi_{x}, c_{0, \ldots, 5}$ etc. Figures 1 and 2 in [75] exhibit the excellent agreement with lattice-QCD data in the interval $T \in[100,500] \mathrm{MeV}$ and remaining uncertainties due to limited precision, in particular of the sound velocity in the interval $T \in[100,160] \mathrm{MeV}$ and the susceptibility $\chi_{4}$. The scale setting is accomplished by $L^{-1}=5.148 \mathrm{GeV} .^{1}$ The locus of the minimum sound velocity squared is described in leading order by

$T_{\min \left\{v_{s}^{2}\right\}}\left(\mu_{B}\right)=T_{v_{s}^{2}}\left(\mu_{B}=0\right)\left[1-\kappa\left(\frac{\mu_{B}}{T_{v_{s}^{2}}\left(\mu_{B}=0\right)}\right)^{2}\right]$,

with $T_{v_{s}^{2}}\left(\mu_{B}=0\right)=145 \mathrm{MeV}$ and $\kappa=0.0178 \mathrm{MeV}$. Note that $T_{v_{s}^{2}}\left(\mu_{B}=0\right)<T_{\mathrm{pc}}$. Despite the direct relation to an observable, the location of $\min \left\{v_{s}^{2}\right\}$ is not so precisely constrained by lattice-QCD data as that of the maximum of chiral susceptibility which determines quite accurately the pseudocritical deconfinement temperature $T_{\mathrm{pc}}\left(\mu_{B}=0\right)$ [32,33]. In so far, the curves $T_{\min \left\{v_{s}^{2}\right\}}\left(\mu_{B}\right)$ and $T_{\mathrm{pc}}\left(\mu_{B}\right)$ need not coincide.

The EdM model with these input data is then ready to transport the thermodynamic information from $\mu_{B}=0$ to $\mu_{B}>0$, thus uncovering the $T-\mu_{B}$ plane. This is very much the spirit of the quasiparticle model [78,79], where a flow equation facilitates such a transport.

\section{SPECTRAL FUNCTIONS}

The equation of motion (3) of $\varphi$ can also be employed to compute quarkonia spectral functions, cf. [38,4749,80,81]. For $\omega^{2}=p^{\mu} p_{\mu}>0$ fixed, the asymptotic boundary behavior facilitates two linearly independent solutions by considering the leading-order terms on both sides of the interval $\left[0, z_{H}\right]:$ (i) for $z \rightarrow 0^{+}$, one has the general solution $\varphi(z \rightarrow 0) \rightarrow A(\omega) \varphi_{1}+B(\omega) \varphi_{2}$, due to the AdS asymptotic at the boundary, with two $\omega$-dependent complex constants $A$ and $B$, and $\varphi_{1}(z \rightarrow 0) \rightarrow 1$ and $\varphi_{2}(z \rightarrow 0) \rightarrow\left(z / z_{H}\right)^{2}$, and (ii) near the horizon, $z \rightarrow z_{H}^{-}$, the asymptotic behavior of solutions of (3) is steered by the poles of $1 / f$ and $1 / f^{2}$. The two linearly independent solutions are $\varphi_{ \pm}\left(z \rightarrow z_{H}\right) \rightarrow\left(1-\frac{z}{z_{H}}\right)^{ \pm i \omega /\left|f^{\prime}\left(z_{H}\right)\right|}$, where $\varphi_{ \pm}$

\footnotetext{
${ }^{1}$ References [64,65] use the dilaton potential $L^{2} V(\phi)=$ $-12 \cosh (\gamma \phi)+\phi_{2} \phi^{2}+\phi_{4} \phi^{4}$ which delivers for $\left(\gamma, \phi_{2}, \phi_{4}\right)=$ $(0.568,-1.92,-0.04)$, i.e., $\Delta^{V}=3.9$ also a good description of lattice-QCD data of $v_{s}^{2}(T)$ by $L^{-1}=1.99 \mathrm{GeV}$. The difference of these scale settings can be traced back to the sensibility of internal model quantities, such as the dilaton profile $\phi\left(z, z_{H}\right)$, while observables remain stable, since effects of different model parametrizations cancel out when considering observables. Note that the parametrization (8) implies the conformal dimension $\Delta^{V}=2.76$.
}

represent out-going and in-falling solutions, respectively. The general near-horizon solution is given by $\left.\varphi\left(z \rightarrow z_{H}\right) \rightarrow C(\omega) \varphi_{(} z\right)++D(\omega) \varphi_{-}(z)$, again with complex constants $C$ and $D$ which depend on $\omega$. The side conditions for the bulk-to-boundary propagator are $\varphi(0)=1$, which means $A(\omega)=1$, and $\varphi\left(z \rightarrow z_{H}\right)=$ $\varphi_{-}\left(z \rightarrow z_{H}\right)$ (purely an in-falling solution at the black hole horizon), yielding $C(\omega)=0$. Due to the bilinear mapping $(A, B) \mapsto(C, D)$, the value of $B$ for getting the desired infalling solution can be determined by solving the above equations twice, once with $A=1, B=0$ and once with $A=0, B=1$, and comparing the result with $\varphi_{-}$to dig out the proper coefficients.

The corresponding retarded Green function $\mathcal{G}^{\mathrm{R}}$ of the dual current operator $\bar{Q} \gamma_{\mu} Q$, defined within the framework of the holographic dictionary via a generating functional by $\mathcal{G}^{\mathrm{R}}=\frac{\delta^{2}}{\delta \mathcal{A}^{0 \mu}(-\omega) \delta \mathcal{A}_{\mu}^{0}(\omega)}\left\langle\exp \left\{i \int \mathrm{d}^{4} x \mathcal{A}_{\nu}^{0} \bar{Q} \gamma^{\nu} Q\right\}\right\rangle$, is given by

$$
\begin{aligned}
\mathcal{G}^{\mathrm{R}}(\omega) & =\frac{\delta^{2} S_{m}^{V, \text { on-shell }}}{\delta \mathcal{A}^{0 \mu}(-\omega) \delta \mathcal{A}_{\mu}^{0}(\omega)}=\frac{1}{k_{V}} \lim _{z \rightarrow 0} \frac{1}{z} \varphi^{*}(z) \varphi^{\prime}(z) \\
& =\frac{2}{k_{V} z_{H}^{2}} B(\omega),
\end{aligned}
$$

with $\mathcal{A}_{\mu}^{0} \equiv \epsilon_{\mu} \exp \left\{i p_{\nu} x^{\nu}\right\}$ for $\mu \in\{1,2,3\}$ [81]. The quantity $S_{m}^{V \text {,on-shell }}$ denotes here the action (1) with the solution $\varphi$ from (3). Finally, the spectral function $\rho$ follows from $\rho(\omega)=\operatorname{Im} \mathcal{G}^{\mathrm{R}}(\omega)=\frac{2}{k_{V} z_{H}^{2}} \operatorname{Im} B(\omega)$. It has the dimension of energy squared, suggesting to use $L^{2} \rho$ or $\rho / \omega^{2}$ as convenient representations.

\section{NUMERICAL RESULTS}

The spectral function $\rho\left(\omega, T, \mu_{B}\right)$ is accessible by numerical means by the following chain of operations: (i) solving the equations of motion (A1)-(A4) following from the action (7) with boundary conditions (A5)-(A10) for the background encoded in $A_{0}(z), f_{0}(z)=1, \phi_{0}(z)$ with the prescribed $V\left(\phi_{0}\right)$ from Eq. (8) yields the input for Eqs. (5) and (6) for the determination of $G_{m}(\phi)$ at $T=0$ (highlighted by the subscript " 0 ," using $U_{0}$ from Appendix B; for parameter values, see Appendix B 2), (ii) using afterwards that $G_{m}(\phi)$ in Eqs. (3) and (11) but with $A\left(z, z_{H}\right), f\left(z, z_{H}\right), \phi\left(z, z_{H}\right)$ determined again by $V(\phi)$ via the equations of motion (A1)-(A4) following from the action (7) with boundary conditions (A5)-(A10) and $\mathcal{F}(\phi)$ from Eq. (9), see Appendix A. Some care is needed in that numerical treatment.

Our results are exhibited in Fig. 1 for the $\Upsilon$ meson. We emphasize that neither an explicit quark-mass dependence enters our approach (instead, quark masses are implicitly accounted for via $U_{0}(z)$ for entering $\left.G_{m}\right)$ nor a confinement criterion (instead, narrow spectral functions as quasiparticle states are considered as confined $J^{\mathrm{PC}}=1^{--} \bar{b} b$ states). In so far, the emergence of such narrow quasiparticle ground 

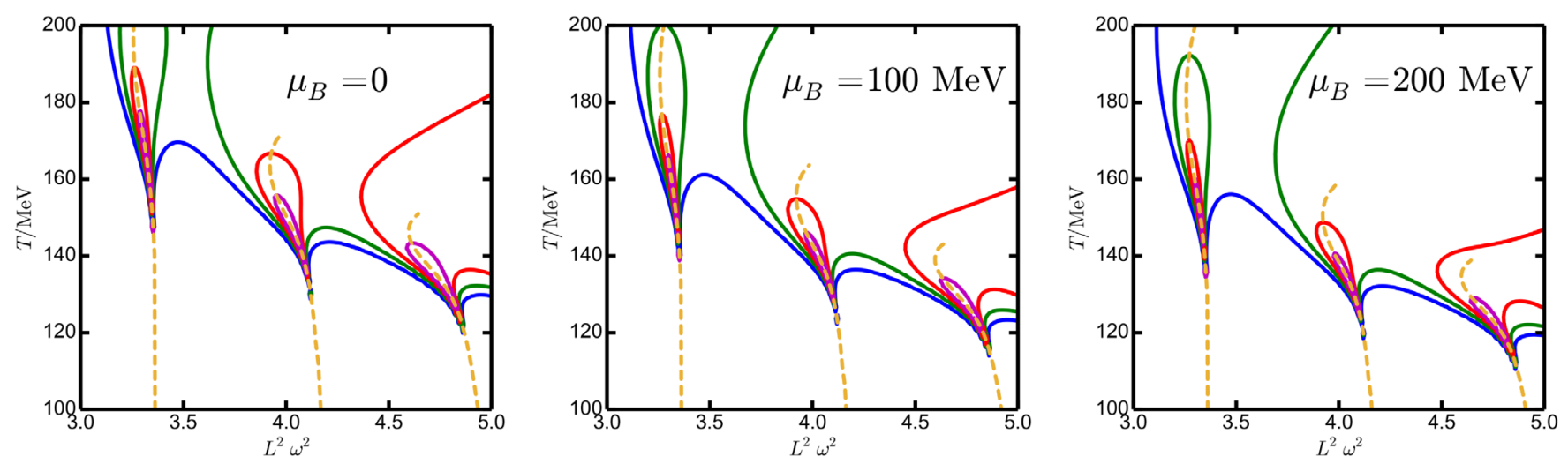

FIG. 1. Contour plots of the $\Upsilon$ spectral function $L^{2} \rho\left(\omega, T, \mu_{B}\right)$ over the temperature vs scaled frequency $L^{2} \omega^{2}$ plane for $\mu_{B}=0$ (left), $100 \mathrm{MeV}$ (middle) and $200 \mathrm{MeV}$ (right); blue, green, red and magenta contour curves correspond to $L^{2} \rho=1,3,10$ and 30 . The dashed orange curves mark the maximum positions of $L^{2} \rho\left(\omega, T, \mu_{B}\right)$ with respect to $\omega$. At large temperatures, such local maxima disappear and the respective state can be considered as completely molten. At lower temperatures, sharp quasiparticles have been formed, i.e., the contours are squeezed and are hidden behind the dashed orange curves.


FIG. 2. $\Upsilon$ spectral function $L^{2} \rho\left(\omega, T, \mu_{B}\right)$ as a function of scaled frequency $L^{2} \omega^{2}$ for $\mu_{B}=0$ (left), $100 \mathrm{MeV}$ (middle) and $200 \mathrm{MeV}$ (right) at $155 \mathrm{MeV}$ (red), $150 \mathrm{MeV}$ (green) and $145 \mathrm{MeV}$ (blue). These plots arise from Fig. 1 as cross sections at constant temperature.

states at a temperature around the deconfinement temperature $T_{\mathrm{pc}}$ is astonishing. The higher the excitation, the later the excited-state formation happens when considering the cooling due to expansion. The net effect of the finite baryochemical potential $\mu_{B}$ is a lowering of the formation temperature.

The density of low mass color carriers increases by a nonzero baryo-chemical potential $\mu_{B}$ or baryon density. For instance, adding simply the leading-order net baryon density term $\propto T^{2} \mu_{B}$ to the total number density at $\mu_{B}=$ 0 (which is $\propto T^{3}$ ), one gets the estimate $\propto T^{3}+[\cdot] T^{2} \mu_{B}$ in leading order of small $\mu_{B}$ and species-dependent positive constant $[\cdot]$. Otherwise, estimating the total number density in a fugacity expansion in massless Stefan-Boltzmann approximation would result in a term $\propto T^{3}$ plus a term $\propto T \mu_{B}^{2}$. In either case of such rough estimates, the number density of color charges increases. In the spirit of the Matsui-Satz conjecture [82], the color charge screening becomes stronger, and therefore the formation of bound states as sharp quasiparticles is delayed (suppressed) in a cooling medium. This is consistent with the finding in the holographic approach [83] with D7-branes embedded in the AdS-Schwarzschild black hole background, where the nonzero baryon density also facilitates a broadening of the probe vector meson spectral function. A closer look at the contour curves at $T=140 \ldots 160 \mathrm{MeV}$ suggests $\rho\left(\omega, T-15 \mathrm{MeV}, \mu_{B}=0\right) \approx \rho\left(\omega, T, \mu_{B}=200 \mathrm{MeV}\right)$, meaning that the $\Upsilon$ formation pattern is shifted down by a temperature of about $15 \mathrm{MeV}$ by the impact of the baryochemical potential $\mu_{B}=200 \mathrm{MeV}$. Otherwise, the minimum sound velocity, $T_{\min \left\{v_{s}^{2}\right\}}\left(\mu_{B}\right)$, drops only by about $5 \mathrm{MeV}$ when going from $\mu_{B}=0$ to $\mu_{B}=200 \mathrm{MeV}$. While being rather semiquantitative and restricted to $\Upsilon$, this finding may be interpreted as a hint to a gap between freeze-out and deconfinement temperature, i.e., $T_{f o}\left(\mu_{B}\right) \neq$ $T_{p c}\left(\mu_{B}\right)$ at $\mu_{B}>0$.

Focusing on the crucial temperature region near $T_{\mathrm{pc}}$ or $T_{v_{s}^{2}}$, one observes how rapidly the ground state evolves toward a sharp quasiparticle within this narrow interval of $T$ at $\mu_{B}=0$, see the left sharp peak in the left panel in Fig. 2. The first excitation (the middle peak) becomes clearly visible, with peak position noticeably shifting up upon 

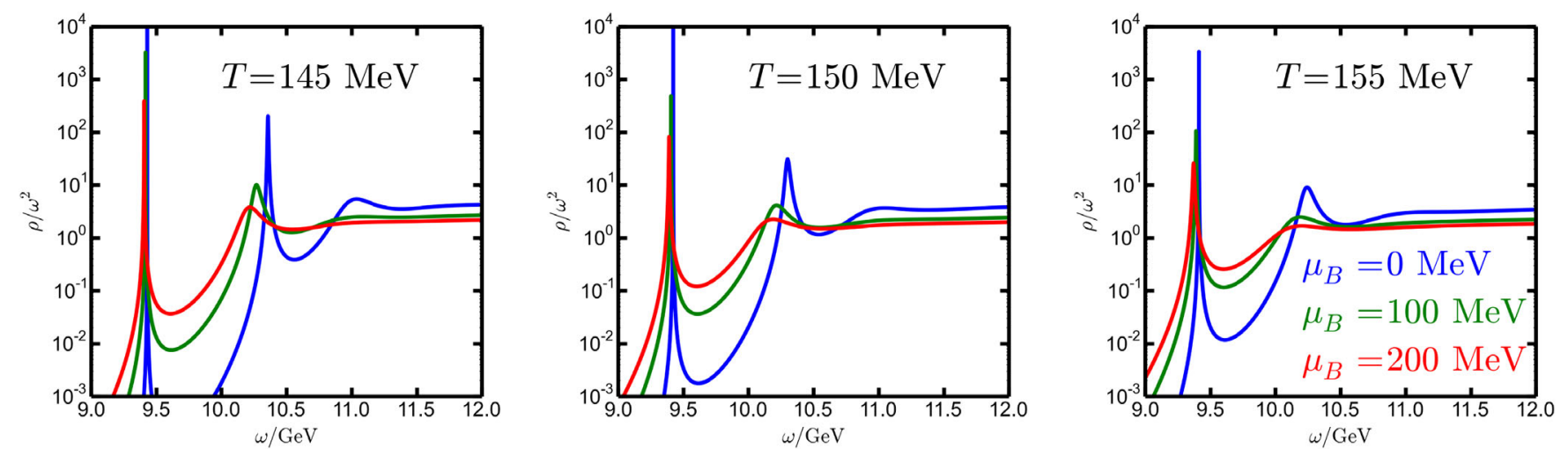

FIG. 3. Scaled $\Upsilon$ spectral function $\rho\left(\omega, T, \mu_{B}\right) / \omega^{2}$ as a function of energy $\omega$ for $T=145 \mathrm{MeV}$ (left, i.e., at $T / T_{v_{s}^{2}}=1$ ), $150 \mathrm{MeV}$ (middle) and $155 \mathrm{MeV}$ (right, i.e., at $T \approx T_{p c}$ ) at $\mu_{B}=0$ (blue), $100 \mathrm{MeV}$ (green) and $200 \mathrm{MeV}$ (red). The data of Fig. 2 is rearranged to highlight the $\mu_{B}$ dependence and to furnish a comparison with experimental results which use $M_{\mu^{+} \mu^{-}}$as abscissa.

dropping temperature. In contrast, the second excitation is identifiable at $T=145 \mathrm{MeV}$ but not so clearly at higher temperatures. These trends continue at $\mu_{B}>0$, see middle and right panels in Fig. 2. At $\mu_{B}=200 \mathrm{MeV}$, the second excitation is not identifiable as a clear peak down to $T=145 \mathrm{MeV}$, while the first excitation sticks out only at $T \leq 150 \mathrm{MeV}$. Let us emphasize that, at $\mu_{B}=0$, the first and (weakly) the second excitations are identifiable as peak structures, in contrast to [52], where these excitations appear as molten, while the ground state persists up to high temperatures since it is kept by a narrow deep well potential.

To highlight the $\mu_{B}$ dependence, we exhibit in Fig. 3 the same spectral functions arranged in reversed order, i.e., various values of $\mu_{B}$ at a given temperature. Such a representation evidences the impact of the baryo-chemical potential in a clear manner. Note that in an adiabatically cooling strong-interaction system one should employ the isentropic curves $T\left(\mu_{B}\right)$ to follow the evolution of the spectral function. Figure 3 provides some guidance for that.

The relation of the spectral function to the resulting $\mu^{+} \mu^{-}$ spectrum from $\Upsilon \rightarrow \mu^{+} \mu^{-}$may be elaborated as in previous studies, e.g., by superimposing the thermal yield (which needs a model of the space-time evolution of the fireball) and the post-freeze-out contribution (which is directly related to the $\Upsilon(n S)$ yields and feedings) and the various background sources. This is beyond the scope of our paper. Nevertheless, the emerging picture of our model (see $\mu_{B}=0$ curves in Fig. 3) appears at first glance qualitatively consistent with experimental observations [1-4]. The strengths of excited states, $\Upsilon(2 S, 3 S)$, are gradually suppressed with respect to the ground state $\Upsilon(1 S)$ in heavy-ion collisions with participant numbers $N_{\text {part }}>100$, most notably the $3 S$ state, while in $p p$ collisions one clearly identifies $\Upsilon(2 S, 3 S)$ as prominent peaks, albeit with decreasing strengths. One could imagine that convoluting our spectral functions with a finite (fiducial) resolution leads to a picture better resembling the observations, e.g., Fig. 1 in [1]. In fact, applying a Gaussian resolution function according to the scheme $\bar{\rho}_{\sigma}(\omega)=\int_{0}^{\infty} \mathrm{d} \bar{\omega} \bar{\rho}(\bar{\omega}) \frac{1}{\sqrt{2 \pi \sigma^{2}}} \exp \left\{-\frac{(\bar{\omega}-\omega)^{2}}{2 \sigma^{2}}\right\} \quad$ with $\quad \bar{\rho}(\omega):=$ $\rho(\omega) / \omega^{2}$ and selecting the ad hoc value $\sigma=0.1 \mathrm{GeV}$ generates a pattern closer to the observation, see Fig. 4.
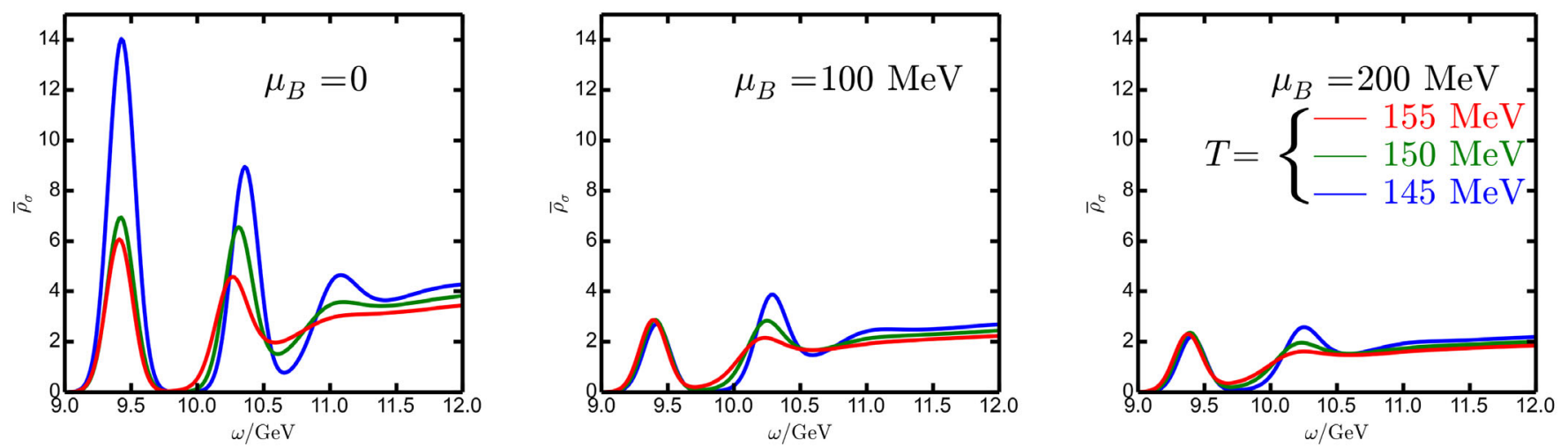

FIG. 4. The same data as in Fig. 2 is used but convoluted with a resolution function with a width of $100 \mathrm{MeV}$ (see the text) to enable a comparison with experimental results which use linear scales. An adaptive $\bar{\omega}$ grid with the minimum spacing of $10^{-8} L$ is employed. Note that feeding is not included. 
While the underlying soft-wall holographic potential captures approximately the mass spectrum of radial excitations at $T=0$, it facilitates, also in the present background, decay constants increasing with (or independent of) the radial quantum number $n$, in contrast to experimental data. This imperfection seems to continue to $T>0$ : the strengths of (i.e., yields from) excitations become too large. Therefore, we do not introduce a continuum or background subtraction, as discussed in [52], and leave further refinements (e.g., the options offered in Appendix B 3 with respect to decay constants) and feeding corrections to follow-up work.

\section{CONCLUSION AND SUMMARY}

Using a bottom-up holographic model with minimalistic field content, we investigate the impact of the finite baryochemical potential $\mu_{B}$ on bottomonium formation at temperatures in the order of the hadron chemical freeze-out in relativistic heavy-ion collisions. The model has two pillars: (i) the vector meson part which employs the bottomonium masses of ground state and radial excitations as input to adjust a suitable Schrödinger-equivalent potential, and (ii) the Einstein-dilaton-Maxwell background which is adjusted independently to lattice-QCD thermodynamics (sound velocity and light-quark susceptibilities). The field content is as follows: (i) a bottomonium-specific function $G_{m}(\phi)$, which encodes implicitly the $b$ quark masses via the Schrödinger-equivalent potential $U_{0}(z)$ and is essential for the bulk-to-boundary propagator $\varphi(z)$, and (ii) the gravity-dilaton-Maxwell part, determined dynamically by the dilaton potential $V(\phi)$ and the dilaton-Maxwell coupling $\mathcal{F}(\phi)$. Since there is neither a confinement criterion nor a chiral condensate as the order parameter in such an approach, we consider the shrinking of the spectral function $\rho$ (determined by $\varphi$ ) to a narrow quasiparticle state as bottomonium formation in a cooling strong-interaction medium. Despite a simple two-parameter Schrödingerequivalent potential $U_{0}$, we find the bottomonium ground state formation at about a temperature of $150 \mathrm{MeV}$ at $\mu_{B}=0$. Increasing $\mu_{B}$ drops the formation temperature. Excited states are consecutively formed at lower temperatures, or the spectral strengths are not yet concentrated completely at the $T=0$ quasiparticle energy at a given temperature. This fits well in the experimental observation that the $\Upsilon(2 S)$ and $\Upsilon(3 S)$ states are hardly identifiable in the dilepton spectra in heavy-ion collisions at LHC, while the ground state is clearly visible [1]. In contrast, $\Upsilon(1 S, 2 S, 3 S)$ are clearly seen in proton-proton collisions at the same beam energies per nucleon.

Our approach assumes rapid thermalization and equilibration, since the cooling of the medium is handled as a sequence of equilibrium states. Off-equilibrium phenomena up to dynamical freeze-out need to be considered in refined investigations. A closer contact to string theory would be highly desirable to overcome the deployed phenomenological parametrizations steering our two pillars, background thermodynamics and the vacuum mass spectrum.

\section{ACKNOWLEDGMENTS}

The authors gratefully acknowledge the collaboration with J. Knaute and thank M. Ammon, P. Braun-Munzinger, R. Critelli, M. Kaminski, J. Noronha, K. Redlich and G. Röpke for useful discussions. The work is supported in part by the European Unions Horizon 2020 research and innovation program STRONG-2020 under Grant agreement No. 824093.

\section{APPENDIX A: USING THE BULK COORDINATE $z$ IN THE EdM MODEL}

The equations of motion for the dilaton $\phi(z)$, the Maxwell field $\Phi(z)$ with the coupling function $\mathcal{F}(\phi)$, following from the action (7), read in the coordinates (2) with warp factor $\exp \{A(z)\}$ and blackening function $f(z)$

$$
\begin{gathered}
\phi^{\prime \prime}=-\left[\frac{3}{2} A^{\prime}+(\log f)^{\prime}\right] \phi^{\prime}-\frac{1}{f}\left[e^{A} \frac{\partial V(\phi)}{\partial \phi}+\frac{1}{8} \frac{\partial \mathcal{F}(\phi)}{\partial \phi} \Phi^{\prime 2}\right], \\
\Phi^{\prime \prime}=-\left[\frac{1}{2} A^{\prime}+\frac{1}{4} \frac{\log \mathcal{F}(\phi)}{\partial \phi} \phi^{\prime}\right] \Phi^{\prime}, \\
A^{\prime \prime}=\frac{1}{2} A^{\prime 2}-\frac{1}{3} \phi^{\prime 2}, \\
f^{\prime \prime}=-\frac{3}{2} A^{\prime} f^{\prime}-\frac{1}{4} \mathcal{F}(\phi) e^{-A} \Phi^{\prime 2} .
\end{gathered}
$$

The leading-order initial conditions are (i) near the boundary, i.e., $z \rightarrow 0^{+}$,

$$
\begin{gathered}
\lim _{z \rightarrow 0} \phi=0, \quad \lim _{z \rightarrow 0} \phi^{\prime}=0, \\
\lim _{z \rightarrow 0} \Phi=\mu_{B} L, \\
\lim _{z \rightarrow 0} A=-2 \log \frac{z}{L}+\cdots, \quad \lim _{z \rightarrow 0} A^{\prime}=-\frac{2}{z}+\cdots, \\
\lim _{z \rightarrow 0} f=1,
\end{gathered}
$$

and (ii) near the horizon, i.e., $z \rightarrow z_{H}^{-}$,

$$
\begin{aligned}
& \lim _{z \rightarrow z_{H}} \Phi=0, \\
& \lim _{z \rightarrow z_{H}} f=0 .
\end{aligned}
$$

Equations (A9) and (A10) make these equations a mixed boundary problem. The Hawking temperature is 
determined by $T\left(z_{H}\right)=-\left.\frac{1}{4 \pi} f\left(z, z_{H}\right)^{\prime}\right|_{z=z_{H}}$ with a freely chosen value of the horizon position $z_{H}$ and free choice of $\mu_{B}$. For completeness, we note also entropy density $s\left(T, \mu_{B}\right)=\frac{2 \pi}{\kappa_{5}^{2}} \exp \left\{\frac{3}{2} A\left(z_{H}\right)\right\}$ and baryon density $n_{B}\left(T, \mu_{B}\right)=$ $-\frac{1}{\kappa_{5}^{2}} \Phi_{2}$ with $\Phi_{2}$ from the small-z expansion $\Phi=$ $\mu_{B} L+\Phi_{2}(z / L)^{2}+\cdots$.

References [73-76] use essentially the coordinates originally employed in $[62,63]$ with special gauging of the radial coordinate. These solutions can be parametrized by the double $\left(\phi\left(z_{H}\right), \Phi\left(z_{H}\right)\right)$ and need a posteriori the determination of the (screwed) $T-\mu_{B}$ mesh. The advantage of Eqs. (A1)-(A10) is in the boundary conditions (A8) and (A9), which make the scan of the $T-\mu_{B}$ plane easier.

\section{APPENDIX B: UV-IR MATCHING}

\section{Generalities}

In the zero-temperature limit, $T \rightarrow 0$ at $\mu_{B}=0$, one has $f \rightarrow 1, \xi \rightarrow z$ and $U \rightarrow U_{0}$ in Eq. (4). The primary request to any useful Schrödinger-equivalent potential $U_{0}(z)$ is to get the proper level spacing via

$$
y^{\prime \prime}-\left(U_{0}-\hat{E} L^{-2}\right) y=0 .
$$

A constant common shift of $U_{0} \rightarrow U_{0}^{b}=U_{0}+4 b / L^{2}$ can be absorbed in $\hat{E} \rightarrow \hat{E}^{b}=\hat{E}+4 b$ to accomplish the wanted meson ground state mass squared, $L^{2} m_{0}^{(b) 2}=\hat{E}_{0}+4 b$, independent of $U_{0}(z)$. Here, we suppose that Eq. (B1) delivers a set of discrete eigenvalues $\hat{E}_{n} \equiv L^{2} m_{n}^{2}, n=$ $0,1,2, \ldots$ by the requirement of square-integrable solutions $y_{n}$.

We emphasize again that $U_{0}(z)$ is an independent input in our approach which determines $\mathcal{S}_{0}$, via $\mathcal{S}_{0}^{\prime 2}+\frac{1}{2} \mathcal{S}_{0}^{2}-$ $2 U_{0}=0$ from Eq. (5), and $G_{m}\left(\phi_{0}\right)$, via $\left(\log G_{m}\right)^{\prime}-\mathcal{S}_{0}+$ $\frac{1}{2} A_{0}^{\prime}-\phi_{0}^{\prime}=0$ from Eq. (6). The needed holographic background quantities $A_{0}(z)$ and $\phi_{0}(z)$ are determined independently by the dilaton potential $V(\phi)=V\left(\phi_{0}\right)$, where the quantities at $T=0$ and $\mu_{B}=0$ are labeled by the subscript " 0 ." The identification $G_{m}(\phi)=G_{m}\left(\phi_{0}\right)$ determines via Eqs. (3) and (11) the spectral function. In so far, the choice of $U_{0}$ deserves some special attention.

\section{Approximately uncovering the $r$ mass spectrum}

The famous soft-wall (SW) model [84] employs $U_{0}^{S W}=$ $U_{0}^{\mathrm{UV}}+U_{0}^{\mathrm{IR}}=\frac{3}{4} z^{-2}+(a / L)^{2}(z / L)^{2}$ with the leadingorder asymptotic parts

$$
\begin{gathered}
\lim _{z \rightarrow 0} U_{0} \rightarrow U_{0}^{\mathrm{UV}}(z):=\frac{\alpha^{2}}{z^{2}}, \quad \alpha^{2} \equiv \frac{3}{4}, \\
\lim _{z \rightarrow \infty} U_{0} \rightarrow U_{0}^{\mathrm{IR}}(z):=\frac{a^{2}}{L^{2}}\left(\frac{z}{L}\right)^{2} .
\end{gathered}
$$

It has one free parameter, $a$, and, in general, cannot accommodate independently the ground state mass and level spacing at the same time. Nevertheless, it delivers via $L^{2} m_{n}^{2}=4 a(n+1), n=0,1,2 \cdots$, the Regge type mass spectrum-in [84], termed "linear confinement." Despite the imperfection, it has been used in [47,48] for an investigation of the thermal behavior of the $J / \psi$ spectral function. Supplemented with the shift parameter $b$, i.e., $U_{0}^{S W} \rightarrow U_{0}^{S W, b}=U_{0}^{S W}+4 b / L^{2}$, however, the ground state mass and uniform level spacing can be tuned separately and may be used as a minimum parameter model with $\hat{E}_{n}^{(b)} \equiv L^{2} m_{n}^{(b) 2}=4 b+4 a(n+1)$. The decay constants are less perfectly reproduced, as stressed in [49] for $J / \psi$ and $\psi^{\prime}$. Nevertheless, due to its transparency we stay with this variant in our study of the $\Upsilon$ spectral function. The parameters $a=0.2006$ and $b=0.6436$, together with the scale setting $L^{-1}=5.148 \mathrm{GeV}$ used in Sec. V, result in $m_{0}^{(b)}=m_{\Upsilon(1 S)}=9.460 \mathrm{GeV}, \quad m_{1}^{(b)}=$ $10.524 \mathrm{GeV} \quad\left(=m_{\Upsilon(2 S)}+5 \%\right) \quad$ and $\quad m_{2}^{(b)}=11.490 \mathrm{GeV}$ $\left(=m_{\Upsilon(3 S)}+11 \%\right)$. A readjustment $a \rightarrow 0.1035$ and $b \rightarrow 0.7407$ would lead also to the exact experimental mass of $m_{1}^{(b)}=m_{\Upsilon(2 S)}=10.0233 \mathrm{GeV}$ as well as $m_{2}^{(b)}=$ $10.556 \mathrm{GeV} \quad\left(=m_{\Upsilon(3 S)}+2 \%\right)$ to be compared to $m_{\Upsilon(3 S)}=10.3553 \mathrm{GeV}$, but reduced somewhat the formation temperature, as discussed in [65]. The dependencies on additional parameters discussed below can be used for finetuning by breaking the uniform level spacing $\hat{E}_{n+1}-\hat{E}_{n}=$ $4 a$ of the soft-wall model.

The relation $a \ll b$ suggests a separation of scales. The level spacing, $m^{2}(\Upsilon((n+1) S))-m^{2}(\Upsilon((n S)) \ll$ $\frac{1}{2}\left(m^{2}(\Upsilon((n+1) S))+m^{2}(\Upsilon((n S)))\right.$ for $n=0,1,2$ may be attributed to QCD dynamics, while the mass gap or average hadron masses squared may be related to the heavy-quark mass. This calls for a separate consideration of the level spacing as part of fine-tuning in Subsec. B 3.

In attempting fine-tuning, one may proceed in a two-step approach by (i) first accomplishing the level spacing only, and (ii) eventually shifting the whole spectrum to accomplish the wanted values of $m_{n}^{(b)}$. Applied to $U_{0}^{S W, b}$, step (i) would fix $a$, and $b$ is obtained in step (ii). While such a two-step fine-tuning procedure looks promising, it could be hampered by a problem which we faced, e.g., in $[64,65]$ : unfavorable parametrizations of $U_{0}(z)$ can lead to formation temperatures that are too low, such that at $T_{\mathrm{pc}}$ quasiparticles are not yet formed, in contrast to the common understanding of hadron formation in relativistic heavy-ion collisions discussed in the introduction. The origin of the affair can be qualitatively explained within the transparent model $U_{0}^{S W, b}$. At finite temperatures, $U_{0}(z) \rightarrow U_{T}\left(z, z_{H}(T)\right)$. Since $U_{T}\left(z, z_{H}\right) \propto$ $f\left(z, z_{H}\right)$ according to Eq. (5), one can imagine $U_{T}\left(z, z_{H}\right) \approx U_{0}(z) \Theta\left(z_{H}-z\right)$. To accommodate the ground state in such a potential, the IR turning point (t.p., in the 
spirit of $\mathrm{WKB}) \quad z^{I R t . p .} \approx 2 \sqrt{a+b} / a$ must obey $z^{I R t . p .}<z_{H} \approx 1 /(4 \pi T)$. In other words, to allow for an "unmolested" state at given temperature $T$, the parameter $a$ must be sufficiently large to get small $z^{\text {IRt.p. }}$. This is the reasoning of considering the quark-mass effect encoded in $m_{0}$ as primary quantity and a less strict parameter adjustment for the level spacing, as deployed in the above parameter setting. (In stark contrast, [85] puts emphasis on the correct decay constants and is less restrictive to the bottomonium mass spectrum with the advantage of rather persistent states up to high temperatures $T>T_{p c}$.)

\section{Fine-tuning of $U_{0}$ to recover $\Upsilon(1 S, 2 S, 3 S)$ masses by proper level spacing}

The asymptotic parts at small $z$ (UV), Eq. (B2), and large $z$ (IR), Eq. (B3), can be joined in many different ways to a common Schrödinger-equivalent potential $U_{0}(z)$ to be used in Eqs. (4) and, in vacuum, (B1) to accomplish the wanted fine-tuning. Here we mention only one with a minimum set of parameters. An easy choice would be

$$
U_{0}^{d i p}=\left\{\begin{array}{l}
U_{0}^{\mathrm{UV}}(z)+b^{\mathrm{UV}} L^{-2} \text { for } z<z_{0}, \\
\tilde{U}_{0} \text { for } z_{0} \leq z \leq \lambda z_{0}, \\
U_{0}^{\mathrm{IR}}(z)+b^{\mathrm{IR}} L^{-2} \text { for } z>\lambda z_{0},
\end{array}\right.
$$

with constant parameters $b^{U V, I R}, \tilde{U}_{0}, \lambda$ and the scale setting parameter $L$. The options $\lambda=1$ (dip related to discontinuity at $x_{0}$ ), $\lambda \rightarrow 1$ (mimicking a Dirac delta dip at $x_{0}$ when $\tilde{U}_{0} \propto 1 /(\lambda-1)$ ) and $\lambda>1$ (boxlike dip within $x_{0} \cdots \lambda x_{0}$ ) with respect to fine-tuning of the mass spectrum are discussed in the Supplemental Material to this article in [86]. We finish this paper with the expectation that the tendency of the $\mu_{B}$ dependence of spectral functions is not obstructed by the details of approximately or accurately adjusting parameters of $U_{0}$ to the $\Upsilon$ mass spectrum.
[1] S. Chatrchyan et al. (CMS Collaboration), Phys. Rev. Lett. 109, 222301 (2012); 120, 199903(E) (2018).

[2] A. M. Sirunyan et al. (CMS Collaboration), Phys. Rev. Lett. 120, 142301 (2018).

[3] A. M. Sirunyan et al. (CMS Collaboration), Phys. Lett. B 790, 270 (2019).

[4] S. Acharya et al. (ALICE Collaboration), Phys. Lett. B 790, 89 (2019).

[5] S. Acharya et al. (ALICE Collaboration), Phys. Lett. B 822 , 136579 (2021).

[6] S. Aronson, E. Borras, B. Odegard, R. Sharma, and I. Vitev, Phys. Lett. B 778, 384 (2018).

[7] X. Du, R. Rapp, and M. He, Phys. Rev. C 96, 054901 (2017).

[8] J. Hoelck, F. Nendzig, and G. Wolschin, Phys. Rev. C 95, 024905 (2017).

[9] G. Wolschin, Int. J. Mod. Phys. A 35, 2030016 (2020).

[10] X. Yao and B. Müller, Phys. Rev. D 100, 014008 (2019).

[11] X. Yao, W. Ke, Y. Xu, S. A. Bass, and B. Müller, J. High Energy Phys. 01 (2021) 046.

[12] X. Yao, W. Ke, Y. Xu, S. A. Bass, and B. Müller, Proc. Sci., HardProbes2020 (2021) 064 [arXiv:2009.05658].

[13] M. Strickland, Proc. Sci., High-pT2019 (2020) 020 [arXiv:1906.00888].

[14] N. Brambilla, M. Escobedo, M. Strickland, A. Vairo, P. Vander Griend, and J. H. Weber, J. High Energy Phys. 05 (2021) 136.

[15] F. Liu, E. Wang, X.-N. Wang, N. Xu, and B.-W. Zhang, Nucl. Phys. A1005, 122081 (2021).

[16] F. Prino and R. Rapp, J. Phys. G 43, 093002 (2016).

[17] R. Rapp et al., Nucl. Phys. A979, 21 (2018).

[18] Y. Xu et al., Phys. Rev. C 99, 014902 (2019).

[19] S. Cao et al., Phys. Rev. C 99, 054907 (2019).
[20] N. Brambilla, M. A. Escobedo, A. Vairo, and P. Vander Griend, Phys. Rev. D 100, 054025 (2019).

[21] T. Song, P. Moreau, J. Aichelin, and E. Bratkovskaya, Phys. Rev. C 101, 044901 (2020).

[22] C. Chattopadhyay and U.W. Heinz, Phys. Lett. B 801, 135158 (2020).

[23] D. Bazow, U. W. Heinz, and M. Strickland, Phys. Rev. C 90, 054910 (2014).

[24] R. Katz and P. B. Gossiaux, Ann. Phys. (Amsterdam) 368, 267 (2016).

[25] J. P. Blaizot and M. A. Escobedo, J. High Energy Phys. 06 (2018) 034.

[26] J. P. Blaizot and M. A. Escobedo, Phys. Rev. D 98, 074007 (2018).

[27] N. Brambilla, M. A. Escobedo, J. Soto, and A. Vairo, Phys. Rev. D 97, 074009 (2018).

[28] A. Rothkopf, Phys. Rep. 858, 1 (2020).

[29] P. Braun-Munzinger and B. Dönigus, Nucl. Phys. A987, 144 (2019).

[30] A. Andronic, P. Braun-Munzinger, K. Redlich, and J. Stachel, Nature (London) 561, 321 (2018).

[31] T. Reichert, G. Inghirami, and M. Bleicher, Eur. Phys. J. A 56, 267 (2020).

[32] A. Bazavov et al. (HotQCD Collaboration), Phys. Lett. B 795, 15 (2019).

[33] S. Borsanyi, Z. Fodor, J. N. Guenther, R. Kara, S. D. Katz, P. Parotto, A. Pasztor, C. Ratti, and K. K. Szabó, Phys. Rev. Lett. 125, 052001 (2020).

[34] S. Borsanyi, Z. Fodor, C. Hoelbling, S. D. Katz, S. Krieg, and K. K. Szabo, Phys. Lett. B 730, 99 (2014).

[35] A. Bazavov et al. (HotQCD Collaboration), Phys. Rev. D 90, 094503 (2014). 
[36] H. Suganuma, T. M. Doi, K. Redlich, and C. Sasaki, J. Phys. G 44, 124001 (2017).

[37] R. Bellwied, J. Noronha-Hostler, P. Parotto, I. Portillo Vazquez, C. Ratti, and J. M. Stafford, Phys. Rev. C 99, 034912 (2019).

[38] P. Colangelo, F. Giannuzzi, and S. Nicotri, J. High Energy Phys. 05 (2012) 076.

[39] P. Colangelo, F. Giannuzzi, and S. Nicotri, Phys. Rev. D 80, 094019 (2009).

[40] P. Colangelo, F. De Fazio, F. Giannuzzi, F. Jugeau, and S. Nicotri, Phys. Rev. D 78, 055009 (2008).

[41] R. Zöllner and B. Kämpfer, Phys. Rev. C 94, 045205 (2016).

[42] R. Zöllner and B. Kämpfer, J. Phys. Conf. Ser. 878, 012023 (2017).

[43] R. Zöllner and B. Kämpfer, J. Phys. Conf. Ser. 1024, 012003 (2018).

[44] N. R. F. Braga and R. Da Mata, Phys. Lett. B 804, 135381 (2020).

[45] N. R. F. Braga, M. A. Martin Contreras, and S. Diles, Europhys. Lett. 115, 31002 (2016).

[46] N. R. F. Braga, M. A. Martin Contreras, and S. Diles, Eur. Phys. J. C 76, 598 (2016).

[47] M. Fujita, K. Fukushima, T. Misumi, and M. Murata, Phys. Rev. D 80, 035001 (2009).

[48] M. Fujita, T. Kikuchi, K. Fukushima, T. Misumi, and M. Murata, Phys. Rev. D 81, 065024 (2010).

[49] H. R. Grigoryan, P. M. Hohler, and M. A. Stephanov, Phys. Rev. D 82, 026005 (2010).

[50] N. R. F. Braga, L. F. Ferreira, and A. Vega, Phys. Lett. B 774, 476 (2017).

[51] N. R. F. Braga and L. F. Ferreira, Phys. Lett. B 773, 313 (2017).

[52] M. A. Martin Contreras, S. Diles, and A. Vega, Phys. Rev. D 103, 086008 (2021).

[53] A. Bazavov, F. Karsch, Y. Maezawa, S. Mukherjee, and P. Petreczky, Phys. Rev. D 91, 054503 (2015).

[54] S. Kim, P. Petreczky, and A. Rothkopf, J. High Energy Phys. 11 (2018) 088.

[55] A. L. Kruse, H. T. Ding, O. Kaczmarek, H. Ohno, and H. Sandmeyer, MDPI Proc. 10, 45 (2019), arXiv:1901.04226.

[56] R. Larsen, S. Meinel, S. Mukherjee, and P. Petreczky, Phys. Lett. B 800, 135119 (2020).

[57] S. Borsanyi et al., Nature (London) 539, 69 (2016).

[58] S. S. Gubser and A. Nellore, Phys. Rev. D 78, 086007 (2008).

[59] S. I. Finazzo, R. Rougemont, H. Marrochio, and J. Noronha, J. High Energy Phys. 02 (2015) 051.

[60] S. I. Finazzo and J. Noronha, Phys. Rev. D 89, 106008 (2014).
[61] R. Zöllner and B. Kämpfer, Eur. Phys. J. Plus 135, 304 (2020).

[62] O. DeWolfe, S. S. Gubser, and C. Rosen, Phys. Rev. D 83, 086005 (2011).

[63] O. DeWolfe, S. S. Gubser, and C. Rosen, Phys. Rev. D 84, 126014 (2011).

[64] R. Zöllner and B. Kämpfer, Eur. Phys. J. Special Topics 229, 3585 (2020).

[65] R. Zöllner and B. Kämpfer, Particles 4, 159 (2021).

[66] G. Odyniec (STAR Collaboration), Proc. Sci., CORFU2018 (2019) 151.

[67] M. Abdallah et al. (STAR Collaboration), Phys. Rev. C 104, 024902 (2021).

[68] A. Bzdak, S. Esumi, V. Koch, J. Liao, M. Stephanov, and N. $\mathrm{Xu}$, Phys. Rep. 853, 1 (2020).

[69] L. Bellantuono, P. Colangelo, F. De Fazio, F. Giannuzzi, and S. Nicotri, Phys. Rev. D 96, 034031 (2017).

[70] X. Yao and B. Müller, Phys. Rev. C 97, 014908 (2018); 97, 049903(E) (2018).

[71] N. R. F. Braga and R. da Mata, Phys. Rev. D 101, 105016 (2020).

[72] R. Rougemont, A. Ficnar, S. Finazzo, and J. Noronha, J. High Energy Phys. 04 (2016) 102.

[73] J. Grefa, J. Noronha, J. Noronha-Hostler, I. Portillo, C. Ratti, and R. Rougemont, Phys. Rev. D 104, 034002 (2021).

[74] R. Critelli, J. Noronha, J. Noronha-Hostler, I. Portillo, C. Ratti, and R. Rougemont, Phys. Rev. D 96, 096026 (2017).

[75] J. Knaute, R. Yaresko, and B. Kämpfer, Phys. Lett. B 778, 419 (2018).

[76] J. Knaute and B. Kämpfer, Phys. Rev. D 96, 106003 (2017).

[77] R. Bellwied, S. Borsanyi, Z. Fodor, S. D. Katz, A. Pasztor, C. Ratti, and K. K. Szabo, Phys. Rev. D 92, 114505 (2015).

[78] A. Peshier, B. Kämpfer, and G. Soff, Phys. Rev. C 61, 045203 (2000).

[79] A. Peshier, B. Kämpfer, and G. Soff, Phys. Rev. D 66, 094003 (2002).

[80] P. M. Hohler and Y. Yin, Phys. Rev. D 88, 086001 (2013).

[81] D. Teaney, Phys. Rev. D 74, 045025 (2006).

[82] T. Matsui and H. Satz, Phys. Lett. B 178, 416 (1986).

[83] J. Erdmenger, M. Kaminski, P. Kerner, and F. Rust, J. High Energy Phys. 11 (2008) 031.

[84] A. Karch, E. Katz, D. T. Son, and M. A. Stephanov, Phys. Rev. D 74, 015005 (2006).

[85] N. R. F. Braga and L. F. Ferreira, J. High Energy Phys. 01 (2019) 082.

[86] R. Zöllner and B. Kämpfer, arXiv:2109.05824. 